\title{
Momentum space approach to crossing symmetric CFT correlators. Part II. General spacetime dimension
}

\author{
Hiroshi Isono, ${ }^{a}$ Toshifumi Noumi ${ }^{b}$ and Gary Shiu ${ }^{c}$ \\ ${ }^{a}$ Department of Physics, Faculty of Science, Chulalongkorn University, \\ Bangkok 10330, Thailand \\ ${ }^{b}$ Department of Physics, Kobe University, \\ Kobe 65\%-8501, Japan \\ ${ }^{c}$ Department of Physics, University of Wisconsin-Madison, \\ Madison, WI 53706, U.S.A. \\ E-mail: hiroshi.isono81@gmail.com, tnoumi@phys.sci.kobe-u.ac.jp, \\ shiu@physics.wisc.edu
}

ABSTRACT: Our previous work [1] constructed, in three-dimensional momentum space, a manifestly crossing symmetric basis for scalar conformal four-point functions, based on the factorization property proposed by Polyakov. This work extends this construction to general dimensional conformal field theory. To facilitate the treatment of symmetric traceless tensors, we exploit techniques of spherical harmonics in general dimensions.

KEYwords: AdS-CFT Correspondence, Conformal Field Theory, Conformal and W Symmetry

ARXIV EPRINT: 1908.04572 


\section{Contents}

1 Introduction 1

2 Properties of spherical harmonics 2

2.1 Spherical harmonics in general dimension and Funk-Hecke formula 3

2.2 Derivation of the Funk-Hecke formula 4

3 Two- and three-point functions 5

3.1 Helicity decomposition of spinning operators 6

$\begin{array}{lll}3.2 & \text { Two-point functions } & 7\end{array}$

3.3 Three-point functions of two scalars and one tensor 8

$\begin{array}{ll}3.4 & \text { Analytic properties }\end{array}$

4 Crossing symmetric basis for scalar four-point functions 11

$\begin{array}{lll}4.1 & \text { Strategy } & 11\end{array}$

$\begin{array}{ll}4.2 \text { Construction of Polyakov block } & 12\end{array}$

5 Conclusion $\quad 14$

$\begin{array}{ll}\text { A Derivation of eq. (3.14) } & 14\end{array}$

$\begin{array}{ll}\text { B Derivation of eq. (3.26) } & 15\end{array}$

\section{Introduction}

The crossing symmetric basis of conformal four-point functions, pioneered by Polyakov in 1974 [2], is based on the following ansatz of the expansion of the four-point functions, ${ }^{1}$

$$
\left\langle O_{1}\left(\boldsymbol{k}_{1}\right) O_{2}\left(\boldsymbol{k}_{2}\right) O_{3}\left(\boldsymbol{k}_{3}\right) O_{4}\left(\boldsymbol{k}_{4}\right)\right\rangle^{\prime}=\sum_{O}\left(W_{O}^{(\mathbf{s})}+W_{O}^{(\mathbf{t})}+W_{O}^{(\mathbf{u})}\right)+(\text { analytic terms }),
$$

where the index $O$ labels the intermediate primary operators. $W_{O}^{(\mathbf{s})}$ is called the Polyakov block in the s-channel. Each block satisfies the consistent factorization in momentum space, just as the on-shell factorization of scattering amplitudes of ordinary field theories. The Polyakov block with external scalar operators was shown in Mellin space to be nothing but the Witten exchange diagram [3, 4]. Our paper [1] showed directly in three-dimensional momentum space that the Witten exchange diagram is a natural consequence of the consistent factorization, and constructed the Polyakov block with an intermediate symmetric traceless operator of general spin.

\footnotetext{
${ }^{1}$ Primed correlators are defined as $\langle\ldots\rangle=(2 \pi)^{d} \delta^{(d)}\left(\sum_{i} \boldsymbol{k}_{i}\right)\langle\ldots\rangle^{\prime}$.
} 
This paper extends the construction in [1] to general dimensions. From a technical point of view, the extension to general dimensions becomes a bit more involved due to the treatment of symmetric traceless tensors of general spins. In the three dimensional case [1], we adopted the helicity representation instead of dealing with explicit vector indices. More concretely, one first fixes one of the momenta in the correlation function by using rotational symmetry without loss of generality. One then decomposes a symmetric traceless tensor in the correlator into irreducible representations of the little group of the fixed momentum. In three-dimension, the little group is $\mathrm{O}(2)$ and the expansion is nothing but the Fourier expansion $[1,5]$. In general dimension, the Fourier expansion is replaced by the expansion in spherical harmonics on the general dimensional unit sphere. In the present paper we elucidate this point in general spacetime dimension to construct a crossing-symmetric basis of scalar four-point functions.

The outline of the rest of this paper is as follows. Section 2 is a brief review of the harmonic analysis needed for our analysis. In section 3 we find the helicity representation of two- and three-point functions with spins, and discuss their analytic properties to define the cubic vertex. In section 4 we construct the crossing symmetric basis of scalar four-point functions. The two appendices present derivations of some integral formulae in section 3.

\section{Properties of spherical harmonics}

This section reviews basic properties of spherical harmonics in general spacetime dimensions. The machinery will play a main role in expanding scalar functions of momenta and polarization vectors which appear in conformal two- and three-point functions. Readers familiar with spherical harmonics in general dimension may jump safely to section 3 after checking the Funk-Hecke formula introduced in section 2.1

SO(2) spherical harmonics. Before going into the general dimensional case, let us consider lower dimensional cases, in which the expansion is achieved easily. As the simplest case, we begin with a scalar function of two unit vectors, $\hat{\boldsymbol{w}}=\left(\hat{w}_{1}, \hat{w}_{2}\right)$ and $\hat{\boldsymbol{z}}=\left(\hat{z}_{1}, \hat{z}_{2}\right)$, in two dimension. More explicitly, it is a function of the inner product of the two unit vectors, $f(\hat{\boldsymbol{w}} \cdot \hat{\boldsymbol{z}})$. If this function is regular at $\hat{\boldsymbol{w}} \cdot \hat{\boldsymbol{z}}=0$, we may expand it as

$$
f(\hat{\boldsymbol{w}} \cdot \hat{\boldsymbol{z}})=\lambda_{0}+\sum_{m=1}^{\infty} \lambda_{m}\left[\left(\hat{w}_{1}+i \hat{w}_{2}\right)^{m}\left(\hat{z}_{1}-i \hat{z}_{2}\right)^{m}+\left(\hat{w}_{1}-i \hat{w}_{2}\right)^{m}\left(\hat{z}_{1}+i \hat{z}_{2}\right)^{m}\right]
$$

where $\lambda_{m}$ are constant parameters. Notice that the functions $\left(\hat{z}_{1} \pm i \hat{z}_{2}\right)^{m}$ are nothing but the $\mathrm{SO}(2)$ spherical harmonics. If we denote a basis of the $\mathrm{SO}(2)$ spherical harmonics by

$$
Y_{0}(\hat{\boldsymbol{z}})=1, \quad Y_{m+}(\hat{\boldsymbol{z}})=\left(\hat{z}_{1}+i \hat{z}_{2}\right)^{m}, \quad Y_{m-}(\hat{\boldsymbol{z}})=\left(\hat{z}_{1}-i \hat{z}_{2}\right)^{m}
$$

we may rewrite eq. (2.1) as

$$
f(\hat{\boldsymbol{w}} \cdot \hat{\boldsymbol{z}})=\sum_{m=0}^{\infty} \lambda_{m} \Pi_{m}(\hat{\boldsymbol{w}}, \hat{\boldsymbol{z}})
$$


where we introduced the projector $\Pi_{m}$ onto the spin $m$ sector as

$$
\begin{aligned}
\Pi_{0}(\hat{\boldsymbol{w}}, \hat{\boldsymbol{z}}) & =Y_{0}(\hat{\boldsymbol{w}}) Y_{0}^{*}(\hat{\boldsymbol{z}}) \\
\Pi_{m}(\hat{\boldsymbol{w}}, \hat{\boldsymbol{z}}) & =Y_{m+}(\hat{\boldsymbol{w}}) Y_{m+}^{*}(\hat{\boldsymbol{z}})+Y_{m-}(\hat{\boldsymbol{w}}) Y_{m-}^{*}(\hat{\boldsymbol{z}}) \quad(m=1,2, \ldots)
\end{aligned}
$$

The expansion is nothing but the Fourier expansion, as is manifest in polar coordinates.

SO(3) spherical harmonics. A similar expansion applies in the three dimensional case. As we physicists are familiar with, any regular scalar function of two unit vectors, $\hat{\boldsymbol{w}}$ and $\hat{\boldsymbol{z}}$, in three dimension may be expanded by the Legendre polynomials $P_{m}(\hat{\boldsymbol{w}} \cdot \hat{\boldsymbol{z}})$. More explicitly, we may write it in the form (2.3) with some $\Pi_{m} \propto P_{m}(\hat{\boldsymbol{w}} \cdot \hat{\boldsymbol{z}})$. Since the addition theorem of the $\mathrm{SO}(3)$ spherical harmonics states that for any orthonormal basis $Y_{m n}$,

$$
P_{m}(\hat{\boldsymbol{w}} \cdot \hat{\boldsymbol{z}}) \propto \sum_{n=-m}^{m} Y_{m n}(\hat{\boldsymbol{w}}) Y_{m n}^{*}(\hat{\boldsymbol{z}}),
$$

we may normalize $\Pi_{m}$ such that

$$
\Pi_{m}(\hat{\boldsymbol{w}}, \hat{\boldsymbol{z}})=\sum_{n=-m}^{m} Y_{m n}(\hat{\boldsymbol{w}}) Y_{m n}^{*}(\hat{\boldsymbol{z}})
$$

which is again nothing but the projector onto the spin $m$ sector.

\subsection{Spherical harmonics in general dimension and Funk-Hecke formula}

We can generalize the expansion of scalar functions with two unit vectors to general dimensions in a straightforward manner. The expansion is called the Funk-Hecke formula [6-8]: any scalar function of two unit vectors, $\hat{\boldsymbol{w}}$ and $\hat{\boldsymbol{z}}$, in $D$ dimension is expanded as

$$
f(\hat{\boldsymbol{w}} \cdot \hat{\boldsymbol{z}})=\sum_{m=0}^{\infty} \lambda_{m} \Pi_{m}(\hat{\boldsymbol{w}}, \hat{\boldsymbol{z}}) \quad \text { with } \quad \Pi_{m}(\hat{\boldsymbol{w}}, \hat{\boldsymbol{z}})=\sum_{n=1}^{\operatorname{dim} \mathbb{Y}_{m}^{D}} Y_{m n}(\hat{\boldsymbol{w}}) Y_{m n}^{*}(\hat{\boldsymbol{z}}),
$$

where $Y_{m n}$ stands for an orthonormal basis of $\mathrm{SO}(D)$ spherical harmonics with total spin $m$ and $\operatorname{dim} \mathbb{Y}_{m}^{D}$ is the dimension of the spin $m$ representation of $\operatorname{SO}(D) .{ }^{2}$ The coefficients $\lambda_{m}$ are evaluated by the integral,

$$
\lambda_{m}=\frac{\operatorname{vol}\left(S^{D-2}\right)}{\operatorname{vol}\left(S^{D-1}\right)} \int_{-1}^{1} d t\left(1-t^{2}\right)^{\frac{D-3}{2}} P_{m}^{(D)}(t) f(t)
$$

\footnotetext{
${ }^{2}$ In more detail, spherical harmonics are elements of the eigenspace $\mathbb{Y}_{m}^{D}$ with eigenvalues $m(m+D-2)$ of the quadratic Casimir operator of $\mathrm{SO}(D)$,

$$
\Omega_{2}=-\frac{1}{2}\left(z^{\mu} \partial^{\nu}-z^{\nu} \partial^{\mu}\right)\left(z_{\mu} \partial_{\nu}-z_{\nu} \partial_{\mu}\right)
$$

where $z^{\mu}$ is a general $D$-component vector satisfying $z^{\mu}=|\boldsymbol{z}| \hat{z}^{\mu}$. Note that $\Omega_{2}$ is independent of $|\boldsymbol{z}|$. The non-negative integer $m$ is the total spin. The dimension of $\mathbb{Y}_{m}^{D}$ is given by

$$
\operatorname{dim} \mathbb{Y}_{m}^{D}=\frac{(m+D-1) !}{m !(D-1) !}-\frac{(m+D-3) !}{(m-2) !(D-1) !} .
$$

See also the footnote in appendix B for the relation to harmonic polynomials. 
where $P_{m}^{(D)}(t)$ is the normalized Gegenbauer polynomial defined by

$$
P_{m}^{(D)}(t):=\frac{C_{m}^{(D / 2-1)}(t)}{C_{m}^{(D / 2-1)}(1)}
$$

with the Gegenbauer polynomial,

$$
C_{m}^{(\alpha)}(t)=\sum_{n=0}^{[m / 2]}(-)^{n} \frac{\Gamma(m-n+\alpha)}{n !(m-2 n) ! \Gamma(\alpha)}(2 t)^{m-2 n}, \quad C_{m}^{(\alpha)}(1)=\frac{(2 \alpha)_{m}}{m !} .
$$

Here $[x]$ is the integer part of $x$ and $(\alpha)_{m}=\Gamma(\alpha+m) / \Gamma(\alpha)$ is the shifted factorial (also known as the Pochhammer symbol). We may also write the projector $\Pi_{m}$ as $^{3}$

$$
\Pi_{m}(\hat{\boldsymbol{w}}, \hat{\boldsymbol{z}})=\operatorname{dim} \mathbb{Y}_{m}^{D} \cdot P_{m}^{(D)}(\hat{\boldsymbol{w}} \cdot \hat{\boldsymbol{z}}) .
$$

This is called the addition theorem of spherical harmonics. In the rest of this section we summarize the basic properties of the spherical harmonics and review the derivation of the Funk-Hecke formula.

\subsection{Derivation of the Funk-Hecke formula}

We begin with the fact that any function on the unit sphere $S_{D-1}$ can be expanded in spherical harmonics,

$$
f(\hat{\boldsymbol{z}})=\sum_{m=0}^{\infty} \sum_{n=1}^{\operatorname{dim} \mathbb{Y}_{m}^{D}} c_{m n} Y_{m n}(\hat{\boldsymbol{z}}) .
$$

The basis spherical harmonics $Y_{m n}$ are orthogonal and normalized as

$$
\left\langle Y_{m n}, Y_{m^{\prime} n^{\prime}}\right\rangle=\delta_{m m^{\prime}} \delta_{n n^{\prime}}
$$

with the inner product defined by

$$
\langle f, g\rangle=\int_{\hat{\boldsymbol{z}}} d \sigma_{D-1} f^{*}(\hat{\boldsymbol{z}}) g(\hat{\boldsymbol{z}}) .
$$

Here the integration measure $d \sigma_{D-1}$ is the standard one on the unit sphere $S_{D-1}$ with the normalization,

$$
\int_{\hat{z}} d \sigma_{D-1} 1=1
$$

We then introduce the projection operator onto the spin $m$ subspace $\mathbb{Y}_{m}^{D}$ as

$$
\Pi_{m}(\hat{\boldsymbol{z}}, \hat{\boldsymbol{w}})=\sum_{n=1}^{\operatorname{dim} \mathbb{Y}_{m}^{D}} Y_{m n}(\hat{\boldsymbol{z}}) Y_{m n}^{*}(\hat{\boldsymbol{w}}) .
$$

In terms of the projection operator, the spin decomposition (2.15) reads

$$
f=\sum_{m=0}^{\infty} f_{m}, \quad f_{m}(\hat{\boldsymbol{z}})=\int_{\hat{\boldsymbol{w}}} d \sigma_{D-1} \Pi_{m}(\hat{\boldsymbol{z}}, \hat{\boldsymbol{w}}) f(\hat{\boldsymbol{w}}) .
$$

\footnotetext{
${ }^{3}$ We will not attach the dimensionality $D$ to the projector $\Pi_{m}$ and the orthonormal basis vectors $Y_{m n}$ as long as it is obvious from the context.
} 
Explicit form of the projector. Let us derive the expression (2.14) of the projector $\Pi_{m}$ exploiting its rotational invariance. For this purpose, we first introduce the little group $\mathrm{O}(D, \hat{\boldsymbol{a}})$ of a unit vector $\hat{\boldsymbol{a}}$ by

$$
\mathrm{O}(D, \hat{\boldsymbol{a}}):=\{A \in \mathrm{O}(D): A \hat{\boldsymbol{a}}=\hat{\boldsymbol{a}}\} .
$$

A function $f(\hat{\boldsymbol{z}})$ on $S_{D-1}$ is called $\mathrm{O}(D, \hat{\boldsymbol{a}})$-invariant when $f(A \hat{\boldsymbol{z}})=f(\hat{\boldsymbol{z}})$ holds for any element $A \in \mathrm{O}(D, \hat{\boldsymbol{a}})$. It is known that any $\mathrm{O}(D, \hat{\boldsymbol{a}})$-invariant spherical harmonic of spin $m$ is proportional to the normalized Gegenbauer polynomial [8],

$$
f(\hat{\boldsymbol{z}}) \in \mathbb{Y}_{m}^{D} \text { is } \mathrm{O}(D, \hat{\boldsymbol{a}}) \text {-invariant } \Longleftrightarrow f(\hat{\boldsymbol{z}})=P_{m}^{(D)}(\hat{\boldsymbol{z}} \cdot \hat{\boldsymbol{a}}) f(\hat{\boldsymbol{a}}) \text { for any } \hat{\boldsymbol{z}} \in S^{D-1}
$$

We will often use this property. As the first example, let us prove the addition theorem (2.14). Notice first that the projector $\Pi_{m}(\hat{\boldsymbol{z}}, \hat{\boldsymbol{w}})$ is $\mathrm{O}(D)$-invariant because the rotated spherical harmonics $Y_{m n}(A \hat{\boldsymbol{z}})(A \in \mathrm{O}(D))$ form another orthonormal basis of spin $m$. In particular, we have $\Pi_{m}(A \hat{\boldsymbol{z}}, \hat{\boldsymbol{w}})=\Pi_{m}(\hat{\boldsymbol{z}}, \hat{\boldsymbol{w}})$ for any little group transformation $A \in \mathrm{O}(D, \hat{\boldsymbol{w}})$. Therefore, if we think of the projector $\Pi_{m}(\hat{\boldsymbol{z}}, \hat{\boldsymbol{w}})$ as a function of $\hat{\boldsymbol{z}}$, it is an $\mathrm{O}(D, \hat{\boldsymbol{w}})$-invariant spherical harmonic of spin $m$. Therefore, it follows from the equivalence (2.22) that for each $m$

$$
\Pi_{m}(\hat{\boldsymbol{z}}, \hat{\boldsymbol{w}})=\Pi_{m}(\hat{\boldsymbol{w}}, \hat{\boldsymbol{w}}) P_{m}^{(D)}(\hat{\boldsymbol{z}} \cdot \hat{\boldsymbol{w}})=\operatorname{dim} \mathbb{Y}_{m}^{D} \cdot P_{m}^{(D)}(\hat{\boldsymbol{z}} \cdot \hat{\boldsymbol{w}}) .
$$

The Funk-Hecke formula. The Funk-Hecke formula (2.8) with (2.11) is also a consequence of the equivalence (2.22). Regarding a scalar function $f(\hat{\boldsymbol{x}} \cdot \hat{\boldsymbol{z}})$ as a function of $\hat{\boldsymbol{z}}$, we may use the spin decomposition (2.20) to write

$$
\begin{aligned}
f(\hat{\boldsymbol{x}} \cdot \hat{\boldsymbol{z}}) & =\sum_{m=0}^{\infty} \int_{\hat{\boldsymbol{w}}} d \sigma_{D-1} \Pi_{m}(\hat{\boldsymbol{z}}, \hat{\boldsymbol{w}}) f(\hat{\boldsymbol{x}} \cdot \hat{\boldsymbol{w}}) \\
& =\sum_{m=0}^{\infty} \operatorname{dim} \mathbb{Y}_{m}^{D} \int_{\hat{\boldsymbol{w}}} d \sigma_{D-1} P_{m}^{(D)}(\hat{\boldsymbol{z}} \cdot \hat{\boldsymbol{w}}) f(\hat{\boldsymbol{x}} \cdot \hat{\boldsymbol{w}}),
\end{aligned}
$$

where we used the addition theorem (2.14). Since each summand is an $\mathrm{O}(D, \hat{\boldsymbol{x}})$-invariant spherical function of $\hat{\boldsymbol{z}}$ of spin $m$, we may apply the equivalence (2.22) to find

$$
\int_{\hat{\boldsymbol{w}}} d \sigma_{D-1} P_{m}^{(D)}(\hat{\boldsymbol{z}} \cdot \hat{\boldsymbol{w}}) f(\hat{\boldsymbol{x}} \cdot \hat{\boldsymbol{w}})=\lambda_{m} P_{m}^{(D)}(\hat{\boldsymbol{z}} \cdot \hat{\boldsymbol{x}}),
$$

where we introduced

$$
\lambda_{m}=\int_{\hat{\boldsymbol{w}}} d \sigma_{D-1} P_{m}^{(D)}(\hat{\boldsymbol{x}} \cdot \hat{\boldsymbol{w}}) f(\hat{\boldsymbol{x}} \cdot \hat{\boldsymbol{w}})=\frac{\operatorname{vol}\left(S^{D-2}\right)}{\operatorname{vol}\left(S^{D-1}\right)} \int_{-1}^{1} d t\left(1-t^{2}\right)^{\frac{D-3}{2}} P_{m}^{(D)}(t) f(t) .
$$

This concludes the proof of the Funk-Hecke formula (2.8) with (2.11).

\section{Two- and three-point functions}

In this section we introduce helicity representation of conformal correlators with symmetric traceless tensors in general spacetime dimension $d$. After elaborating on the helicity 
decomposition of spinning operators, we utilize the Funk-Hecke formula to derive helicity representation of two- and three-point functions in momentum space. ${ }^{4}$ We then discuss their analytic properties. The cubic vertices introduced there will be used in the next section to construct the crossing symmetric basis.

\subsection{Helicity decomposition of spinning operators}

A standard technique to handle a symmetric traceless tensor in CFT is to contract all vector indices in the tensor operator with a null vector $\epsilon$ called the polarization vector $[45,46] .{ }^{5}$ More explicitly, we denote this in a shorthand notation,

$$
\epsilon^{s} . O=\epsilon^{\mu_{1}} \epsilon^{\mu_{2}} \ldots \epsilon^{\mu_{s}} O_{\mu_{1} \mu_{2} \ldots \mu_{s}},
$$

where $s$ is the spin of the operator $O$. In momentum space it is convenient to further decompose the operator by analogy with the helicity decomposition of massless on-shell particles. Without loss of generality, let us use rotational invariance to set

$$
\boldsymbol{k}=(\mathbf{0}, k),
$$

where $\mathbf{0}$ is the $(d-1)$-component zero vector. It is then convenient to parameterize the polarization vector as

$$
\boldsymbol{\epsilon}=(\hat{\boldsymbol{z}}, i),
$$

where $\hat{\boldsymbol{z}}$ is a $(d-1)$-component real unit vector. With this parameterization the contracted tensor operator $\epsilon^{s}$.O can be thought of as a scalar function on the unit sphere $S^{d-2}$ with the coordinate $\hat{\boldsymbol{z}}$, so that we may decompose it into the helicity operators $O_{m n}$ as

$$
\epsilon^{s} \cdot O(\boldsymbol{k})=\sum_{m=0}^{s} \sum_{n=1}^{\operatorname{dim} \mathbb{Y}_{m}^{d-1}} Y_{m n}(\hat{\boldsymbol{z}}) O_{m n}(\boldsymbol{k}) \quad \text { with } \quad O_{m n}(\boldsymbol{k})=\int_{\hat{\boldsymbol{z}}} d \sigma_{d-2} Y_{m n}^{*}(\hat{\boldsymbol{z}}) \epsilon^{s} \cdot O(\boldsymbol{k}),
$$

where $\left\{Y_{m n}\right\}$ is an orthogonal normal basis for the space of spin $m$ spherical harmonics $\mathbb{Y}_{m}^{d-1}$ on $S^{d-2}$. As explained in section 2.1, this is the decomposition with respect to the spin $m$ of the little group $\mathrm{O}(d-1)$ of $\boldsymbol{k}$ : each spin $m$ sector gives an irreducible representation of the little group. For later use it is convenient to introduce its conjugate as

$$
\overline{O_{m n}}(-\boldsymbol{k})=\int_{\hat{\boldsymbol{z}}} d \sigma_{d-2} \bar{\epsilon}^{s} \cdot O(-\boldsymbol{k}) Y_{m n}(\hat{\boldsymbol{z}}) \quad \text { with } \quad \overline{\boldsymbol{\epsilon}}=(\hat{\boldsymbol{z}},-i),
$$

where note that what is complex conjugate to $O_{m n}(\boldsymbol{k})$ is not $\overline{O_{m n}}(\boldsymbol{k})$, but rather $\overline{O_{m n}}(-\boldsymbol{k})$. Helicity operators with a general momentum are defined in a similar way by performing an appropriate rotation.

\footnotetext{
${ }^{4}$ See, e.g., [5, 9-44] for related developments on conformal correlators in momentum space.

${ }^{5}$ We use the bold and ordinary fonts for vectors and their components, e.g., $\epsilon$ and $\epsilon^{\mu}$. Here $\mu$ is the vector index.
} 


\subsection{Two-point functions}

In momentum space, two-point functions of primary operators with general spins read $[1]^{6}$

$$
\left\langle\epsilon_{1}^{s} \cdot O(\boldsymbol{k}) \bar{\epsilon}_{2}^{s} \cdot O(-\boldsymbol{k})\right\rangle^{\prime}=C_{O O}\left(k^{2}\right)^{\nu}\left[-\frac{\left(\boldsymbol{\epsilon}_{1} \cdot \boldsymbol{k}\right)\left(\overline{\boldsymbol{\epsilon}}_{2} \cdot \boldsymbol{k}\right)}{k^{2}}\right]^{s} P_{s}^{\left(\nu-s, \frac{d}{2}-2\right)}\left(1-\frac{k^{2}\left(\boldsymbol{\epsilon}_{1} \cdot \overline{\boldsymbol{\epsilon}}_{2}\right)}{\left(\boldsymbol{\epsilon}_{1} \cdot \boldsymbol{k}\right)\left(\overline{\boldsymbol{\epsilon}}_{2} \cdot \boldsymbol{k}\right)}\right),
$$

where primed correlators are defined by dropping the delta function for momentum conservation as $\langle\ldots\rangle=(2 \pi)^{d} \delta^{(d)}\left(\sum_{i} \boldsymbol{k}_{i}\right)\langle\ldots\rangle^{\prime}$. We also introduced $\nu=\Delta-\frac{d}{2}$ with the scaling dimension $\Delta$. In this paper we use $\Delta$ and $\nu+\frac{d}{2}$ interchangeably to simplify equations. $P_{n}^{(\alpha, \beta)}$ is the Jacobi polynomial defined by

$$
P_{n}^{(\alpha, \beta)}(t)=\frac{\Gamma(\alpha+n+1)}{n ! \Gamma(\alpha+\beta+n+1)} \sum_{m=0}^{n}\left(\begin{array}{c}
n \\
m
\end{array}\right) \frac{\Gamma(\alpha+\beta+n+m+1)}{\Gamma(\alpha+m+1)}\left(\frac{t-1}{2}\right)^{m} .
$$

Following the last subsection, let us use rotational invariance to set the momentum and the polarization vectors as

$$
\boldsymbol{k}=(\mathbf{0}, k), \quad \boldsymbol{\epsilon}_{1}=\left(\hat{\boldsymbol{z}}_{1}, i\right), \quad \overline{\boldsymbol{\epsilon}}_{2}=\left(\hat{\boldsymbol{z}}_{2},-i\right),
$$

where $\hat{\boldsymbol{z}}_{1}, \hat{\boldsymbol{z}}_{2}$ are $(d-1)$-component real unit vectors. The two-point function then becomes

$$
\left\langle\epsilon_{1}^{s} \cdot O(\boldsymbol{k}) \bar{\epsilon}_{2}^{s} \cdot O(-\boldsymbol{k})\right\rangle^{\prime}=C_{O O}\left(k^{2}\right)^{\nu}(-)^{s} P_{s}^{\left(\nu-s, \frac{d}{2}-2\right)}\left(-\hat{\boldsymbol{z}}_{1} \cdot \hat{\boldsymbol{z}}_{2}\right),
$$

which is a function of $\hat{\boldsymbol{z}}_{1} \cdot \hat{\boldsymbol{z}}_{2}$ multiplied by a helicity-independent factor.

Helicity representation. We then introduce the helicity representation of the two-point functions. Since the two-point function (3.11) is a scalar function of the inner product $\hat{\boldsymbol{z}}_{1} \cdot \hat{\boldsymbol{z}}_{2}$, we may apply the Funk-Hecke formula (2.8) to find

$$
\begin{aligned}
\left\langle\epsilon_{1}^{s} . O(\boldsymbol{k}) \bar{\epsilon}_{2}^{s} . O(-\boldsymbol{k})\right\rangle^{\prime} & =C_{O O}\left(k^{2}\right)^{\nu} \sum_{m=0}^{s} a_{\nu, s}(m) \Pi_{m}\left(\hat{\boldsymbol{z}}_{1}, \hat{\boldsymbol{z}}_{2}\right) \\
& =C_{O O}\left(k^{2}\right)^{\nu} \sum_{m=0}^{s} a_{\nu, s}(m) \sum_{n=1}^{\operatorname{dim} \mathbb{Y}_{m}^{d-1}} Y_{m n}\left(\hat{\boldsymbol{z}}_{1}\right) Y_{m n}^{*}\left(\hat{\boldsymbol{z}}_{2}\right),
\end{aligned}
$$

where $\Pi_{m}\left(\hat{\boldsymbol{z}}_{1}, \hat{\boldsymbol{z}}_{2}\right)$ is the projector (2.19) onto the spin $m$ sector $\mathbb{Y}_{m}^{d-1}$ on the unit sphere $S_{d-2}$. The factor $a_{\nu, s}(m)$ is given by the integral formula (2.11) as

$$
a_{\nu, s}(m)=(-)^{s} \frac{\operatorname{vol}\left(S^{d-3}\right)}{\operatorname{vol}\left(S^{d-2}\right)} \int_{-1}^{1} d t\left(1-t^{2}\right)^{\frac{d}{2}-2} P_{m}^{(d-1)}(t) P_{s}^{\left(\nu-s, \frac{d}{2}-2\right)}(-t),
$$

\footnotetext{
${ }^{6}$ The normalization of the position space correlator is given by

$$
\left\langle\epsilon_{1}^{s} \cdot O\left(\boldsymbol{x}_{1}\right) \bar{\epsilon}_{2}^{s} \cdot O\left(\boldsymbol{x}_{2}\right)\right\rangle=\widetilde{C}_{O O} \frac{\left[\left(\boldsymbol{\epsilon}_{1} \cdot \overline{\boldsymbol{\epsilon}}_{2}\right) x_{12}^{2}-2\left(\boldsymbol{\epsilon}_{1} \cdot \boldsymbol{x}_{12}\right)\left(\overline{\boldsymbol{\epsilon}}_{2} \cdot \boldsymbol{x}_{12}\right)\right]^{s}}{\left(x_{12}^{2}\right)^{\Delta+s}},
$$

which is related to our momentum space normalization as

$$
C_{O O}=2^{s-2 \nu} \pi^{\frac{d}{2}} \frac{s ! \Gamma(-\nu)}{\Gamma\left(\nu+s+\frac{d}{2}\right)} \widetilde{C}_{O O} .
$$


which is computed in appendix A to find

$$
a_{\nu, s}(m)=\frac{2^{d-3} \Gamma\left(\frac{d-1}{2}\right) \Gamma\left(\frac{d}{2}+s-1\right)}{\sqrt{\pi}(s-m) !(s+m+d-3) !} \frac{\Gamma(d+s-\Delta-1) \Gamma(\Delta+m-1)}{\Gamma(d+m-\Delta-1) \Gamma(\Delta-1)} .
$$

The two-point function of helicity operators now reads

$$
\left\langle O_{m n}(\boldsymbol{k}) \overline{O_{m^{\prime} n^{\prime}}}(-\boldsymbol{k})\right\rangle^{\prime}=\delta_{m m^{\prime}} \delta_{n n^{\prime}} C_{O O} a_{\nu, s}(m)\left(k^{2}\right)^{\nu} .
$$

\subsection{Three-point functions of two scalars and one tensor}

We then move on to three-point functions involving two primary scalars and one primary tensor. In momentum space they are given by [1]

$$
\left\langle O_{1}\left(\boldsymbol{k}_{1}\right) O_{2}\left(\boldsymbol{k}_{2}\right) \epsilon^{s} \cdot O\left(\boldsymbol{k}_{3}\right)\right\rangle^{\prime}=\sum_{a=0}^{s}\left(\boldsymbol{\epsilon} \cdot \boldsymbol{k}_{2}\right)^{s-a}\left(\boldsymbol{\epsilon} \cdot \boldsymbol{k}_{3}\right)^{a} V_{12 O}^{(a)}\left(k_{1}, k_{2}, k_{3}\right),
$$

where we collected a helicity-independent part into the last factor as

$$
\begin{aligned}
& V_{12 O}^{(a)}\left(k_{1}, k_{2}, k_{3}\right) \\
& =C_{12 O} \frac{s !}{a !(s-a) !} \frac{\left(\frac{\Delta+s+\mathcal{D}_{12 O}}{2}-a\right)_{a}}{(\Delta-1+s-a)_{a}} \int_{0}^{\infty} \frac{d z}{z^{d+1}} z^{s} \mathcal{B}_{\nu_{1}}\left(k_{1} ; z\right) \mathcal{B}_{\nu_{2}}\left(k_{2} ; z\right) \mathcal{B}_{\nu}\left(k_{3} ; z\right) .
\end{aligned}
$$

Here $C_{12 O}$ is a normalization factor and $\mathcal{B}_{\nu}$ is defined by

$$
\mathcal{B}_{\nu}(k ; z)=\frac{1}{2^{\nu-1} \Gamma(\nu)} k^{\nu} z^{d / 2} K_{\nu}\left(k_{3} z\right)
$$

with $K_{\nu}(z)$ being the modified Bessel function of the second kind. Note that $\mathcal{B}_{\nu}$ is nothing but the bulk-to-boundary propagator of a scalar field on $\operatorname{AdS}_{d+1}$ with mass $m^{2}=\nu^{2}-d^{2} / 4$. Also $\mathfrak{D}_{12 O}$ is a differential operator defined by $\left(k_{12}:=\left|\boldsymbol{k}_{1}+\boldsymbol{k}_{2}\right|\right)$

$$
\mathfrak{D}_{12 O}=\frac{k_{1}^{2}-k_{2}^{2}}{k_{12}^{2}}\left(k_{1} \partial_{k_{1}}+k_{2} \partial_{k_{2}}-\Delta_{t}+s+2 d\right)-\left[\left(k_{1} \partial_{k_{1}}-\Delta_{1}\right)-\left(k_{2} \partial_{k_{2}}-\Delta_{2}\right)\right] \text {. }
$$

We refer the reader to ref. [1] for details of the helicity independent part (3.17). In the following we instead discuss the helicity structure of the three-point function (3.16).

Helicity representation. As in the case of two-point functions, we use rotational invariance to fix the momentum of the tensor $O$ and parameterize the polarization vector as

$$
\boldsymbol{k}_{3}=\left(\mathbf{0}, k_{3}\right), \quad \boldsymbol{\epsilon}=(\hat{\boldsymbol{z}}, i),
$$

where $\hat{\boldsymbol{z}}$ is a $(d-1)$-component real unit vector as before. With this parameterization the three-point function (3.16) reads

$$
\left\langle O_{1}\left(\boldsymbol{k}_{1}\right) O_{2}\left(\boldsymbol{k}_{2}\right) \epsilon^{s} . O\left(\boldsymbol{k}_{3}\right)\right\rangle^{\prime}=\sum_{a=0}^{s}\left[k_{2}^{s-a}\left(i k_{3}\right)^{a}\left(i \cos \theta+\hat{\boldsymbol{\kappa}}_{2} \cdot \hat{\boldsymbol{z}} \sin \theta\right)^{s-a}\right] V_{12 O}^{(a)},
$$


where we parameterized the momentum $\boldsymbol{k}_{2}$ with the $(d-1)$-component unit vector $\hat{\boldsymbol{\kappa}}_{2}$ as

$$
\boldsymbol{k}_{2}=k_{2}\left(\hat{\boldsymbol{\kappa}}_{2} \sin \theta, \cos \theta\right)
$$

We then decompose the three-point function (3.21) in the spin of the little group of $\boldsymbol{k}_{3}$. Since this is a scalar function of the inner product $\hat{\boldsymbol{\kappa}}_{2} \cdot \hat{\boldsymbol{z}}$, the Funk-Hecke formula (2.8) yields the following spin decomposition:

$$
\begin{aligned}
\left\langle O_{1}\left(\boldsymbol{k}_{1}\right) O_{2}\left(\boldsymbol{k}_{2}\right) \epsilon^{s} \cdot O\left(\boldsymbol{k}_{3}\right)\right\rangle^{\prime} & =\sum_{m=0}^{s} V_{12 O}(m, \theta) \Pi_{m}^{d-1}\left(\hat{\boldsymbol{\kappa}}_{2}, \hat{\boldsymbol{z}}\right) \\
& =\sum_{m=0}^{s} V_{12 O}(m, \theta) \sum_{n=1}^{\operatorname{dim} \mathbb{Y}_{m}^{d-1}} Y_{m n}^{*}\left(\hat{\boldsymbol{\kappa}}_{2}\right) Y_{m n}(\hat{\boldsymbol{z}})
\end{aligned}
$$

In other words the three-point function with the helicity operator $O_{m n}$ is given by

$$
\left\langle O_{1}\left(\boldsymbol{k}_{1}\right) O_{2}\left(\boldsymbol{k}_{2}\right) O_{m n}\left(\boldsymbol{k}_{3}\right)\right\rangle^{\prime}=V_{12 O}(m, \theta) Y_{m n}^{*}\left(\hat{\boldsymbol{\kappa}}_{2}\right) .
$$

Here the $m$-dependent factor $V_{12 O}(m, \theta)$ is given through the integral formula $(2.11)$ by

$$
\begin{aligned}
V_{12 O}(m, \theta)= & \sum_{a=0}^{s} V_{12 O}^{(a)} k_{2}^{s-a}\left(i k_{3}\right)^{a} \\
& \times \frac{\operatorname{vol}\left(S^{d-3}\right)}{\operatorname{vol}\left(S^{d-2}\right)} \int_{-1}^{1} d t\left(1-t^{2}\right)^{\frac{d}{2}-2} P_{m}^{(d-1)}(t)\left[(i \cos \theta+t \sin \theta)^{s-a}\right] .
\end{aligned}
$$

In appendix B we derive its analytic expression as

$$
V_{12 O}(m, \theta)=\sum_{a=0}^{s-m} V_{12 O}^{(a)} k_{2}^{s-a} k_{3}^{a} \frac{i^{s-m}(s-a) !}{2^{m}(s-a-m) !\left(\frac{d-1}{2}\right)_{m}} \sin ^{m} \theta \cdot \widehat{P}_{s-a, m}^{(d)}(\cos \theta),
$$

where note that the summation is over $0 \leq a \leq s-m$. Here we introduced $\widehat{P}_{s-a, m}^{(d)}(t):=$ $P_{s-a-m}^{(d+2 m)}(t)$. For $d=3$, the combination $\sin ^{m} \theta \cdot \widehat{P}_{s-a, m}^{(d)}(\cos \theta)$ is proportional to the associated Legendre function in particular.

\subsection{Analytic properties}

At the end of this section, we discuss analytic properties of three-point functions. In particular, we demonstrate that the non-analytic part of a three-point function enjoys a factorization similarly to scattering amplitudes. Our argument here is parallel to that in ref. [1], to which we refer the reader for more detailed explanations.

To discuss analytic properties, let us first rearrange eq. (3.24) into the form,

$$
\begin{aligned}
\left\langle O_{1}\left(\boldsymbol{k}_{1}\right) O_{2}\left(\boldsymbol{k}_{2}\right) O_{m n}\left(\boldsymbol{k}_{3}\right)\right\rangle^{\prime}= & \left(k_{2} \sin \theta\right)^{m} Y_{m n}^{*}\left(\hat{\boldsymbol{\kappa}}_{2}\right) \\
& \times \mathcal{A}_{12 O}^{(m)}\left(\boldsymbol{k}_{1}, \boldsymbol{k}_{2}, \boldsymbol{k}_{3} ; \mathfrak{D}_{12 O}\right) \int_{0}^{\infty} \frac{d z}{z^{d+1}} z^{s} \mathcal{B}_{\nu_{1}}\left(k_{1} ; z\right) \mathcal{B}_{\nu_{2}}\left(k_{2} ; z\right) \mathcal{B}_{\nu}\left(k_{3} ; z\right),
\end{aligned}
$$


where we introduced a differential operator $\mathcal{A}_{12 O}^{(m)}$ as

$$
\begin{aligned}
\mathcal{A}_{12 O}^{(m)}\left(\boldsymbol{k}_{1}, \boldsymbol{k}_{2}, \boldsymbol{k}_{3} ; \mathfrak{D}_{12 O}\right)= & C_{12 O} \frac{i^{s-m} s !}{2^{m}\left(\frac{d-1}{2}\right)_{m}(s-m) !} \\
& \times \sum_{a=0}^{s-m} \frac{(s-m) !}{a !(s-m-a) !} k_{2}^{s-m-a} \widehat{P}_{s-a, m}^{(d)}(\cos \theta) k_{3}^{a} \frac{\left(\frac{\Delta+s+\mathfrak{D}_{12 O}}{2}-a\right)_{a}}{(\Delta-1+s-a)_{a}} .
\end{aligned}
$$

Notice that $\mathcal{A}_{12 O}^{(m)}$ depends on the helicity only through the spin $m$ of the little group. An important observation here is that the only source of non-analyticity in the three-point function (3.27) is the integral of three bulk-to-boundary propagators $\mathcal{B}_{\nu}(k ; z)$ : first, the spherical harmonics and the normalized Gegenbauer polynomial are accompanied by an appropriate power in $k_{2}$ as $\left(k_{2} \sin \theta\right)^{m} Y_{m n}^{*}\left(\hat{\boldsymbol{\kappa}}_{2}\right)$ and $k_{2}^{s-m-a} \widehat{P}_{s-a, m}^{(d)}(\cos \theta)$, so that they are polynomials in $\boldsymbol{k}_{2}$. Second, the differential operator $\mathfrak{D}_{12 O}$ always appears in the form $k_{3} \mathfrak{D}_{12 O}$, which is a polynomial in the momenta $\boldsymbol{k}_{i}$ and the Euler operators $k_{i} \partial_{k_{i}}$ because $\left(\boldsymbol{k}_{2}-\boldsymbol{k}_{1}\right) \cdot \boldsymbol{k}_{3} / k_{3}$ is a component of the vector $\boldsymbol{k}_{2}-\boldsymbol{k}_{1}$ along the $\boldsymbol{k}_{3}$ direction. Since the Euler operator does not introduce any new non-analyticity, we conclude that the only source of non-analyticity is the integral in eq. (3.27).

As we mentioned, the integrand of eq. (3.27) contains three bulk-to-boundary propagators $\mathcal{B}_{\nu}(k ; z)$. By looking at analytic properties of $\mathcal{B}_{\nu}(k ; z)$, we find that the integral enjoys the following factorization (see section 3 of ref. [1] for details):

$$
\begin{aligned}
& \operatorname{Disc}_{k_{3}^{2}} \int_{0}^{\infty} \frac{d z}{z^{d+1}} z^{s} \mathcal{B}_{\nu_{1}}\left(k_{1} ; z\right) \mathcal{B}_{\nu_{2}}\left(k_{2} ; z\right) \mathcal{B}_{\nu_{3}}\left(k_{3} ; z\right) \\
& =-\frac{\Gamma\left(1-\nu_{3}\right)}{2^{\nu_{3}}} \int_{0}^{\infty} \frac{d z}{z^{d+1}} z^{s} \mathcal{B}_{\nu_{1}}\left(k_{1} ; z\right) \mathcal{B}_{\nu_{2}}\left(k_{2} ; z\right) z^{d / 2} k_{3}^{-\nu_{3}} I_{\nu_{3}}\left(k_{3} z\right) \times \operatorname{Disc}_{k_{3}^{2}}\left(k_{3}^{2}\right)^{\nu_{3}}
\end{aligned}
$$

where $\operatorname{Disc}_{z}$ denotes a discontinuity on the complex $z$ plane. Also, $I_{\nu}(z)$ is the modified Bessel function of the first kind. Since the prefactor and the differential operator $\mathcal{A}_{12 O}^{(m)}$ in eq. (3.27) do not generate any new non-analyticity as discussed above, the non-analytic part of the three-point function (3.27) also factorizes as

$$
\operatorname{Disc}_{k_{3}^{2}}\left\langle O_{1}\left(\boldsymbol{k}_{1}\right) O_{2}\left(\boldsymbol{k}_{2}\right) O_{m n}\left(\boldsymbol{k}_{3}\right)\right\rangle^{\prime}=T_{12 ; O_{m n}}\left(\boldsymbol{k}_{1}, \boldsymbol{k}_{2} ; \boldsymbol{k}_{3}\right) \operatorname{Disc}_{k_{3}^{2}}\left\langle\overline{O_{m n}}\left(-\boldsymbol{k}_{3}\right) O_{m n}\left(\boldsymbol{k}_{3}\right)\right\rangle^{\prime}
$$

where we introduced what we call the cubic vertex $T_{12 ; O_{m n}}$ as

$$
\begin{aligned}
T_{12 ; O_{m n}}\left(\boldsymbol{k}_{1}, \boldsymbol{k}_{2} ; \boldsymbol{k}_{3}\right) & =\left(k_{2} \sin \theta\right)^{m} Y_{m n}^{*}\left(\hat{\boldsymbol{\kappa}}_{2}\right) \\
& \times \frac{-\Gamma\left(1-\nu_{3}\right)}{2^{\nu_{3}}} \frac{\mathcal{A}_{12 O}^{(m)}}{C_{O O} a_{s, \nu_{3}}(m)} \int_{0}^{\infty} \frac{d z}{z^{d+1}} \mathcal{B}_{\nu_{1}}\left(k_{1} ; z\right) \mathcal{B}_{\nu_{2}}\left(k_{2} ; z\right) z^{d / 2} k_{3}^{-\nu_{3}} I_{\nu_{3}}\left(k_{3} z\right) .
\end{aligned}
$$

Notice that the cubic vertex is analytic at $k_{3}=0$. We will use it in the next section to construct a crossing symmetric basis for scalar four-point functions.

Three-point functions with a conjugate operator. For later use, it is convenient to write down three-point functions involving the conjugate operator $\overline{O_{m n}}$ explicitly. Let us first recall that

$$
\left\langle O_{1}\left(-\boldsymbol{k}_{1}\right) O_{2}\left(-\boldsymbol{k}_{2}\right) \overline{O_{m n}}\left(-\boldsymbol{k}_{3}\right)\right\rangle^{\prime}=\left(\left\langle O_{1}\left(\boldsymbol{k}_{1}\right) O_{2}\left(\boldsymbol{k}_{2}\right) O_{m n}\left(\boldsymbol{k}_{3}\right)\right\rangle^{\prime}\right)^{*} .
$$


Correspondingly, the factorization (3.30) has a conjugate counterpart,

$$
\begin{aligned}
\operatorname{Disc}_{k_{3}^{2}} & \left\langle O_{1}\left(-\boldsymbol{k}_{1}\right) O_{2}\left(-\boldsymbol{k}_{2}\right) \overline{O_{m n}}\left(-\boldsymbol{k}_{3}\right)\right\rangle^{\prime} \\
= & \left(T_{12 ; O_{m n}}\left(\boldsymbol{k}_{1}, \boldsymbol{k}_{2} ; \boldsymbol{k}_{3}\right) \operatorname{Disc}_{k_{3}^{2}}\left\langle\overline{O_{m n}}\left(-\boldsymbol{k}_{3}\right) O_{m n}\left(\boldsymbol{k}_{3}\right)\right\rangle^{\prime}\right)^{*} \\
= & \overline{T_{12 ; O_{m n}}}\left(-\boldsymbol{k}_{1},-\boldsymbol{k}_{2} ;-\boldsymbol{k}_{3}\right) \operatorname{Disc}_{k_{3}^{2}}\left\langle O_{m n}\left(\boldsymbol{k}_{3}\right) \overline{O_{m n}}\left(-\boldsymbol{k}_{3}\right)\right\rangle^{\prime},
\end{aligned}
$$

where we introduced the conjugate cubic vertex $\overline{T_{12 ; O_{m n}}}$ as

$$
\begin{aligned}
& \overline{T_{12 ; O_{m n}}}\left(-\boldsymbol{k}_{1},-\boldsymbol{k}_{2} ;-\boldsymbol{k}_{3}\right) \\
:= & \left(T_{12 ; O_{m n}}\left(\boldsymbol{k}_{1}, \boldsymbol{k}_{2} ; \boldsymbol{k}_{3}\right)\right)^{*} \\
= & \left(k_{2} \sin \theta\right)^{m} Y_{m n}\left(\hat{\boldsymbol{\kappa}}_{2}\right) \frac{-\Gamma\left(1-\nu_{3}\right)}{2^{\nu_{3}}} \\
& \times \frac{\left(\mathcal{A}_{12 O}^{(m)}\left(\boldsymbol{k}_{1}, \boldsymbol{k}_{2}, \boldsymbol{k}_{3} ; \mathfrak{D}_{12 O}\right)\right)^{*}}{C_{O O} a_{s, \nu_{3}}(m)} \int_{0}^{\infty} \frac{d z}{z^{d+1}} \mathcal{B}_{\nu_{1}}\left(k_{1} ; z\right) \mathcal{B}_{\nu_{2}}\left(k_{2} ; z\right) z^{d / 2} k_{3}^{-\nu_{3}} I_{\nu_{3}}\left(k_{3} z\right) .
\end{aligned}
$$

Note that $\theta$ and $\hat{\boldsymbol{\kappa}}_{2}$ are defined in eq. (3.22) in the frame satisfying eq. (3.20).

\section{Crossing symmetric basis for scalar four-point functions}

We then construct the crossing symmetric basis for scalar four-point functions using the ingredients introduced in the previous section. After reviewing the strategy employed in our previous work [1], we provide an explicit construction in general dimension $d$.

\subsection{Strategy}

In the previous section we have demonstrated that the non-analytic part of three-point functions factorizes into the cubic vertex (3.32) and the two-point function as

$$
\operatorname{Disc}_{k_{3}^{2}}\left\langle O_{1}\left(\boldsymbol{k}_{1}\right) O_{2}\left(\boldsymbol{k}_{2}\right) O_{m n}\left(\boldsymbol{k}_{3}\right)\right\rangle^{\prime}=T_{12 ; O_{m n}}\left(\boldsymbol{k}_{1}, \boldsymbol{k}_{2} ; \boldsymbol{k}_{3}\right) \operatorname{Disc}_{k_{3}^{2}}\left\langle\overline{O_{m n}}\left(-\boldsymbol{k}_{3}\right) O_{m n}\left(\boldsymbol{k}_{3}\right)\right\rangle^{\prime},
$$

which is analogous to on-shell factorization of scattering amplitudes. ${ }^{7}$ Its conjugate counterpart is given in eq. (3.33) with the conjugate cubic vertex (3.34). Similarly, we require that the non-analytic part of four-point functions factorizes as

$$
\begin{aligned}
& \operatorname{Disc}_{\mathbf{s}}\left\langle O_{1}\left(\boldsymbol{k}_{1}\right) O_{2}\left(\boldsymbol{k}_{2}\right) O_{3}\left(\boldsymbol{k}_{3}\right) O_{4}\left(\boldsymbol{k}_{4}\right)\right\rangle^{\prime} \\
& =\sum_{O} \sum_{m, n} T_{12 ; O_{m n}}\left(\boldsymbol{k}_{1}, \boldsymbol{k}_{2} ;-\boldsymbol{k}_{12}\right) \operatorname{Disc}_{\mathbf{s}}\left\langle\overline{O_{m n}}\left(\boldsymbol{k}_{12}\right) O_{m n}\left(-\boldsymbol{k}_{12}\right)\right\rangle^{\prime} \overline{T_{34 ; O_{m n}}}\left(\boldsymbol{k}_{3}, \boldsymbol{k}_{4} ; \boldsymbol{k}_{12}\right),
\end{aligned}
$$

where the first sum is over all the intermediate primary operators and the second is over helicity components $O_{m n}$ of the operator $O$. We also introduced $\boldsymbol{k}_{i j}=\boldsymbol{k}_{i}+\boldsymbol{k}_{j}$ and the Mandelstam type variables,

$$
\mathbf{s}=-\left(\boldsymbol{k}_{1}+\boldsymbol{k}_{2}\right)^{2}, \quad \mathbf{t}=-\left(\boldsymbol{k}_{1}+\boldsymbol{k}_{3}\right)^{2}, \quad \mathbf{u}=-\left(\boldsymbol{k}_{1}+\boldsymbol{k}_{4}\right)^{2} .
$$

\footnotetext{
${ }^{7}$ Note that three-point functions enjoy factorization because our correlators are not amputated.
} 
Since the on-shell conditions are not imposed on the external momenta, these variables are independent in contrast to the scattering amplitude case. We require similar factorization in the other channels as well.

Based on the factorization property, we introduce a crossing symmetric basis for conformal four-point functions as [2]

$$
\left\langle O_{1}\left(\boldsymbol{k}_{1}\right) O_{2}\left(\boldsymbol{k}_{2}\right) O_{3}\left(\boldsymbol{k}_{3}\right) O_{4}\left(\boldsymbol{k}_{4}\right)\right\rangle^{\prime}=\sum_{O}\left(W_{O}^{(\mathbf{s})}+W_{O}^{(\mathbf{t})}+W_{O}^{(\mathbf{u})}\right)+(\text { analytic terms }),
$$

where the second term stands for analytic terms which cannot be determined only from analyticity. ${ }^{8}$ The label $O$ again runs over all the intermediate primary operators. The function $W_{O}^{(\mathbf{s})}$, which we call the s-channel Polyakov block, is a conformally covariant function enjoying the following two properties:

1. $W_{O}^{(\mathbf{s})}$ reproduces the s-channel factorization:

$$
\begin{aligned}
\operatorname{Disc}_{\mathbf{s}} W_{O}^{(\mathbf{s})}= & \sum_{m, n} T_{12 ; O_{m n}}\left(\boldsymbol{k}_{1}, \boldsymbol{k}_{2} ;-\boldsymbol{k}_{12}\right) \\
& \times \operatorname{Disc}_{\mathbf{s}}\left\langle\overline{O_{m n}}\left(\boldsymbol{k}_{12}\right) O_{m n}\left(-\boldsymbol{k}_{12}\right)\right\rangle^{\prime} \overline{T_{34 ; O_{m n}}}\left(\boldsymbol{k}_{3}, \boldsymbol{k}_{4} ; \boldsymbol{k}_{12}\right) .
\end{aligned}
$$

2. $W_{O}^{(\mathbf{s})}$ has no non-analyticity other than the one required by s-channel factorization. In particular, it is analytic in $\boldsymbol{k}_{13}$ and $\boldsymbol{k}_{14}$, so free from $\mathbf{t}, \mathbf{u}$-channel discontinuity.

Also, $W_{O}^{(\mathbf{t})}$ and $W_{O}^{(\mathbf{u})}$ are $\mathbf{t}, \mathbf{u}$-channel analogues of $W_{O}^{(\mathbf{s})}$ and enjoy similar properties. This basis manifests the crossing symmetry whereas the consistency with the OPE is obscured, hence the latter provides a nontrivial constraint on the theory [2, 24].

\subsection{Construction of Polyakov block}

Let us proceed to constructing the s-channel Polyakov block. In our previous work [1] we have shown that the Polyakov block with an intermediate scalar operator is nothing but the scalar-exchange Witten diagram: ${ }^{9}$

$$
\begin{aligned}
W_{O}^{(\mathbf{s})}= & C_{12 O} C_{34 O} \int_{0}^{\infty} \frac{d z_{1}}{z_{1}^{d+1}} \int_{0}^{\infty} \frac{d z_{2}}{z_{2}^{d+1}} \\
& \times \mathcal{B}_{\nu_{1}}\left(k_{1} ; z_{1}\right) \mathcal{B}_{\nu_{2}}\left(k_{2} ; z_{1}\right) \mathcal{G}_{\nu_{O}}\left(k_{12} ; z_{1}, z_{2}\right) \mathcal{B}_{\nu_{3}}\left(k_{3} ; z_{2}\right) \mathcal{B}_{\nu_{4}}\left(k_{4} ; z_{2}\right)
\end{aligned}
$$

where we introduced the bulk-to-bulk propagator of the would-be dual bulk scalar as

$$
\begin{aligned}
\mathcal{G}_{\nu}\left(k ; z_{1}, z_{2}\right)= & \frac{\Gamma(1-\nu) \Gamma(1-\nu)}{C_{O O} 2^{2 \nu}} z_{1}^{d / 2} z_{2}^{d / 2}\left[I_{\nu}\left(k z_{1}\right) I_{\nu}\left(k z_{2}\right)\right. \\
& \left.-\theta\left(z_{1}-z_{2}\right) I_{-\nu}\left(k z_{1}\right) I_{\nu}\left(k z_{2}\right)-\theta\left(z_{2}-z_{1}\right) I_{\nu}\left(k z_{1}\right) I_{-\nu}\left(k z_{2}\right)\right] \\
= & \frac{-\Gamma(1-\nu)}{C_{O O} 2^{2 \nu-1} \Gamma(\nu)}\left[\theta\left(z_{1}-z_{2}\right) z_{1}^{d / 2} z_{2}^{d / 2} K_{\nu}\left(k z_{1}\right) I_{\nu}\left(k z_{2}\right)+(1 \leftrightarrow 2)\right] .
\end{aligned}
$$

\footnotetext{
${ }^{8}$ To constrain the analytic contributions, other ingredients will be necessary such as consistency with OPE or locality of the dual bulk theory. Note that the analytic terms correspond to bulk contact terms.

${ }^{9}$ See $[3,4]$ for construction in the Mellin space.
} 
The non-analytic part of the bulk-to-bulk propagator, i.e., the first line of eq. (4.7), is responsible for the factorization so that the first criterion (4.5) of the Polyakov block is satisfied. Furthermore, the last expression of eq. (4.7) guarantees that the bulk-to-bulk propagator exponentially damps down for large $z_{i}$, hence there appear no undesirable singularities, e.g., in the collinear limit $k_{1}+k_{2}=k_{12}$. These criteria specify the form of the Polyakov block up to analytic terms, which correspond to bulk contact interactions. See [1] for more details.

The key observation for extending the construction for intermediate scalars to general spinning operators is that the differential operators,

$$
\mathcal{A}_{12 O}^{(m)}\left(\boldsymbol{k}_{1}, \boldsymbol{k}_{2},-\boldsymbol{k}_{12} ; \mathfrak{D}_{12 O}\right) \text { and }\left(\mathcal{A}_{34 O}^{(m)}\left(-\boldsymbol{k}_{3},-\boldsymbol{k}_{4},-\boldsymbol{k}_{12} ; \mathfrak{D}_{34 O}\right)\right)^{*}
$$

appearing in the cubic vertices (3.32) and (3.34), do not change the non-analytic properties. As a result, we may easily arrive at

$$
\begin{aligned}
W_{O}^{(\mathbf{s})}= & \sum_{m, n}\left(k_{2} \sin \theta_{2} \cdot k_{4} \sin \theta_{4}\right)^{m} Y_{m n}^{*}\left(\hat{\boldsymbol{\kappa}}_{2}\right) Y_{m n}\left(\hat{\boldsymbol{\kappa}}_{4}\right) \\
& \times \frac{\mathcal{A}_{12 O}^{(m)}\left(\boldsymbol{k}_{1}, \boldsymbol{k}_{2} ;-\boldsymbol{k}_{12} ; \mathfrak{D}_{12 O}\right)\left(\mathcal{A}_{34 O}^{(m)}\left(-\boldsymbol{k}_{3},-\boldsymbol{k}_{4},-\boldsymbol{k}_{12} ; \mathfrak{D}_{34 O}\right)\right)^{*}}{a_{s, \nu_{O}}(m)} \\
& \times \int_{0}^{\infty} \frac{d z_{1}}{z_{1}^{d+1-s}} \int_{0}^{\infty} \frac{d z_{2}}{z_{2}^{d+1-s}} \mathcal{B}_{\nu_{1}}\left(k_{1} ; z_{1}\right) \mathcal{B}_{\nu_{2}}\left(k_{2} ; z_{1}\right) \mathcal{G}_{\nu_{O}}\left(k_{12} ; z_{1}, z_{2}\right) \mathcal{B}_{\nu_{3}}\left(k_{3} ; z_{2}\right) \mathcal{B}_{\nu_{4}}\left(k_{4} ; z_{2}\right),
\end{aligned}
$$

where $\mathcal{G}_{\nu_{O}}\left(k_{12} ; z_{1}, z_{2}\right)$ is the scalar bulk-to-bulk propagator defined by eq. (4.7). We also defined the angles $\theta_{2,4}$ and the unit vectors $\hat{\boldsymbol{\kappa}}_{2,4}$ in the frame $-\boldsymbol{k}_{12}=\left(\mathbf{0}, k_{12}\right)$ such that ${ }^{10}$

$$
\boldsymbol{k}_{2}=k_{2}\left(\hat{\boldsymbol{\kappa}}_{2} \sin \theta_{2}, \cos \theta_{2}\right), \quad-\boldsymbol{k}_{4}=k_{4}\left(\hat{\boldsymbol{\kappa}}_{4} \sin \theta_{4}, \cos \theta_{4}\right) .
$$

Again, the non-analytic part of the propagator is responsible for the s-channel factorization and its large $z_{i}$ behavior guarantees that there are no undesirable singularities.

Furthermore, since in eq. (4.9) the spherical harmonics in the first line are the only $n$-dependent factors, the sum over $n$ is nothing but the addition theorem (2.14) with (2.19). The result is

$$
\begin{aligned}
W_{O}^{(\mathbf{s})}= & \sum_{m=0}^{s} \operatorname{dim} \mathbb{Y}_{m}^{d-1} \cdot\left(k_{2} \sin \theta_{2} \cdot k_{4} \sin \theta_{4}\right)^{m} P_{m}^{(d-1)}\left(\hat{\boldsymbol{\kappa}}_{2} \cdot \hat{\boldsymbol{\kappa}}_{4}\right) \\
& \times \frac{\mathcal{A}_{12 O}^{(m)}\left(\boldsymbol{k}_{1}, \boldsymbol{k}_{2} ;-\boldsymbol{k}_{12} ; \mathfrak{D}_{12 O}\right)\left(\mathcal{A}_{34 O}^{(m)}\left(-\boldsymbol{k}_{3},-\boldsymbol{k}_{4},-\boldsymbol{k}_{12} ; \mathfrak{D}_{34 O}\right)\right)^{*}}{a_{s, \nu_{O}}(m)} \\
& \times \int_{0}^{\infty} \frac{d z_{1}}{z_{1}^{d+1-s}} \int_{0}^{\infty} \frac{d z_{2}}{z_{2}^{d+1-s}} \mathcal{B}_{\nu_{1}}\left(k_{1} ; z_{1}\right) \mathcal{B}_{\nu_{2}}\left(k_{2} ; z_{1}\right) \mathcal{G}_{\nu_{O}}\left(k_{12} ; z_{1}, z_{2}\right) \mathcal{B}_{\nu_{3}}\left(k_{3} ; z_{2}\right) \mathcal{B}_{\nu_{4}}\left(k_{4} ; z_{2}\right) .
\end{aligned}
$$

This concludes our construction of the s-channel Polyakov block with an intermediate operator of an arbitrary spin $s$. The $\mathbf{t}, \mathbf{u}$-channel blocks are defined in a similar fashion.

\footnotetext{
${ }^{10}$ Our definition of $\theta_{4}$ and $\hat{\kappa}_{4}$ is motivated by the fact that $\overline{T_{34 ; O_{m n}}}\left(\boldsymbol{k}_{3}, \boldsymbol{k}_{4} ; \boldsymbol{k}_{12}\right)$ is conjugate to $T_{34 ; O_{m n}}\left(-\boldsymbol{k}_{3},-\boldsymbol{k}_{4} ;-\boldsymbol{k}_{12}\right)$.
} 


\section{Conclusion}

This paper generalized the construction of the crossing symmetric basis of scalar fourpoint functions in three spacetime dimension [1] to general dimensions. To deal with the complication due to spins in general spacetime dimension, we utilized techniques of spherical harmonics.

A natural direction to explore along the line of the present work and our previous one [1] is to generalize the construction to correlators involving external spinning operators such as the energy-momentum tensor and other conserved currents. A first step in this direction is the construction of three-point functions with conserved currents and one primary operator of arbitrary spin, which has been done recently in ref. [40]. There will be no conceptual obstruction to constructing the crossing symmetric basis of four-point functions with external conserved currents based on the results there.

Another interesting direction is the extension to de Sitter and inflationary correlators. Some related recent works include [37, 42, 43]. For example, ref. [37] constructed a crossing symmetric basis of de Sitter four-point functions with external scalars of the conformal mass in four dimensions (dual to scalar operators of conformal weight $\Delta=2$ in three dimensions) by solving the conformal Ward-Takahashi identities and studying (non)analytic properties of de Sitter correlators. The extension to four-point functions with external massless scalars was also explored there aiming at applications to inflationary physics. More recently, refs. [42, 43] developed a Mellin representation of exchange diagrams on (anti-)de Sitter space in momentum space for external scalars with arbitrary mass. It would be interesting to explore the relation of these works with ours. We hope to revisit these issues in the near future.

\section{Acknowledgments}

We would like to thank Toshiaki Takeuchi for useful discussion and related collaborations. $\mathrm{HI}$ is supported by the "CUniverse" research promotion project by Chulalongkorn University (grant reference CUAASC). TN is supported in part by JSPS KAKENHI Grant Numbers JP17H02894 and JP18K13539, and MEXT KAKENHI Grant Number JP18H04352. GS is supported in part by the DOE grant DE-SC0017647 and the Kellett Award of the University of Wisconsin.

\section{A Derivation of eq. (3.14)}

We derive the analytic expression (3.14) of the factor $a_{\nu, s}(m)$. We present the integral form of $a_{\nu, s}(m)$ again,

$$
a_{\nu, s}(m)=(-)^{s} \frac{\operatorname{vol}\left(S^{d-3}\right)}{\operatorname{vol}\left(S^{d-2}\right)} \int_{-1}^{1} d t\left(1-t^{2}\right)^{\frac{d}{2}-2} P_{m}^{(d-1)}(t) P_{s}^{\left(\nu-s, \frac{d}{2}-2\right)}(-t) .
$$

To evaluate this, it is convenient to notice that the Gegenbauer polynomial is a special case of the Jacobi polynomial:

$$
C_{m}^{(\lambda)}(t)=(-)^{m} C_{m}^{(\lambda)}(-t)=\frac{(2 \lambda)_{m}}{\left(\lambda+\frac{1}{2}\right)_{m}} P_{m}^{\left(\lambda-\frac{1}{2}, \lambda-\frac{1}{2}\right)}(t)
$$


which may be rephrased in terms of the normalized Gegenbauer polynomial (2.12) as

$$
P_{m}^{(D)}(t)=(-)^{m} P_{m}^{(D)}(-t)=\frac{m !}{\left(\frac{D-1}{2}\right)_{m}} P_{m}^{\left(\frac{D-3}{2}, \frac{D-3}{2}\right)}(t) .
$$

By using the integral formula $(7.391 .10$, p. $807,[47]),{ }^{11}$

$$
\begin{aligned}
& \int_{-1}^{1} d t(1-t)^{\alpha}(1+t)^{\beta} P_{m}^{(\alpha, \beta)}(t) P_{n}^{(\gamma, \beta)}(t) \\
& \quad=\frac{2^{\alpha+\beta+1} \Gamma(\gamma+\beta+n+1+m) \Gamma(\gamma-\alpha+n-m) \Gamma(\alpha+m+1) \Gamma(\beta+n+1)}{m !(n-m) ! \Gamma(\gamma+\beta+n+1) \Gamma(\gamma-\alpha) \Gamma(\alpha+\beta+m+n+2)},
\end{aligned}
$$

we obtain

$$
a_{\nu, s}(m)=\frac{2^{d-3} \Gamma\left(\frac{d-1}{2}\right) \Gamma\left(\frac{d}{2}+s-1\right)}{\sqrt{\pi}(s-m) !(s+m+d-3) !} \frac{\Gamma(d+s-\Delta-1) \Gamma(\Delta+m-1)}{\Gamma(d+m-\Delta-1) \Gamma(\Delta-1)} .
$$

\section{B Derivation of eq. (3.26)}

In this appendix we derive eq. (3.26) by performing the integral (3.25). We first replace the normalized Gegenbauer polynomial in the integral (3.25) by its Rodrigues formula

$$
P_{m}^{(d-1)}(t)=\frac{(-1)^{m}}{2^{m}\left(\frac{d}{2}-1\right)_{m}}\left(1-t^{2}\right)^{2-\frac{d}{2}} \frac{d^{m}}{d t^{m}}\left(1-t^{2}\right)^{m+\frac{d}{2}-2},
$$

and integrate the resulting integral by part $m$ times. Then the integral in the second line of eq. (3.25) becomes

$$
\frac{\operatorname{vol}\left(S^{d-3}\right)}{\operatorname{vol}\left(S^{d-2}\right)} \frac{i^{s-a-m}(s-a) ! \sin ^{m} \theta}{2^{m}\left(\frac{d}{2}-1\right)_{m}(s-a-m) !} \int_{-1}^{1} d t\left(1-t^{2}\right)^{m+\frac{d}{2}-2}(\cos \theta-i t \sin \theta)^{s-a-m} .
$$

Note that it vanishes when $s-a-m<0$. Applying to this integral formula,

$$
P_{m}^{(D)}(\cos \theta)=\frac{\operatorname{vol}\left(S^{D-3}\right)}{\operatorname{vol}\left(S^{D-2}\right)} \int_{-1}^{1} d t\left(1-t^{2}\right)^{\frac{D-4}{2}}(\cos \theta-i t \sin \theta)^{m}
$$

of the normalized Gegenbauer polynomial derived shortly, we find the result (3.26).

Integral formula of the normalized Gegenbauer polynomial. Here we give a derivation of the integral formula (B.3), which had already been used in [1]. For this, we start from the following integral

$$
p(\boldsymbol{z}):=\int_{\hat{\boldsymbol{x}}} d \sigma_{D-2}\left(z_{D}-i \hat{\boldsymbol{x}} \cdot \boldsymbol{z}_{(D-1)}\right)^{m},
$$

where $\boldsymbol{z}$ is a $D$-component vector given by $\boldsymbol{z}=\left(\boldsymbol{z}_{(D-1)}, z_{D}\right)$ with a $(D-1)$-component vector $\boldsymbol{z}_{(D-1)}$ and $\hat{\boldsymbol{x}}$ is a $(D-1)$-component unit vector. Since the integrand $\left(z_{D}-i \hat{\boldsymbol{x}}\right.$. $\left.\boldsymbol{z}_{(D-1)}\right)^{m}$ is harmonic in $\boldsymbol{z}$, its restriction to $S^{D-1}$, namely $p(\hat{\boldsymbol{z}})$, is a spherical harmonic

\footnotetext{
${ }^{11}$ This formula is found in the seventh edition of [47]. A caveat is that the formula in some older editions has a typo.
} 
of spin $m .{ }^{12}$ Furthermore, this is $\mathrm{O}\left(D, \hat{\boldsymbol{e}}_{D}\right)$-invariant, where $\hat{\boldsymbol{e}}_{D}=(\mathbf{0}, 1)$ with $(D-1)$ component zero vector $\mathbf{0}$. We may therefore apply the theorem (2.22) to the spherical harmonic $p(\hat{\boldsymbol{z}})$ of spin $m$, to find

$$
p(\hat{\boldsymbol{z}})=P_{m}^{(D)}\left(\hat{\boldsymbol{z}} \cdot \hat{\boldsymbol{e}}_{D}\right),
$$

where the overall normalization factor is 1 because $p\left(\hat{\boldsymbol{e}}_{D}\right)=1$. Combining eq. (B.5) with eq. (B.4), we find the desired integral representation of the normalized Gegenbauer polynomial,

$$
P_{m}^{(D)}(\cos \theta)=\frac{\operatorname{vol}\left(S^{D-3}\right)}{\operatorname{vol}\left(S^{D-2}\right)} \int_{-1}^{1} d t\left(1-t^{2}\right)^{\frac{D-4}{2}}(\cos \theta-i t \sin \theta)^{m}
$$

where we introduced the angle $\theta$ by $z_{D}=|\boldsymbol{z}| \cos \theta$.

Open Access. This article is distributed under the terms of the Creative Commons Attribution License (CC-BY 4.0), which permits any use, distribution and reproduction in any medium, provided the original author(s) and source are credited.

\section{References}

[1] H. Isono, T. Noumi and G. Shiu, Momentum space approach to crossing symmetric CFT correlators, JHEP 07 (2018) 136 [arXiv:1805.11107] [INSPIRE].

[2] A.M. Polyakov, Nonhamiltonian approach to conformal quantum field theory, Zh. Eksp. Teor. Fiz. 66 (1974) 23 [INSPIRE].

[3] R. Gopakumar, A. Kaviraj, K. Sen and A. Sinha, Conformal Bootstrap in Mellin Space, Phys. Rev. Lett. 118 (2017) 081601 [arXiv:1609.00572] [InSPIRE].

[4] R. Gopakumar, A. Kaviraj, K. Sen and A. Sinha, A Mellin space approach to the conformal bootstrap, JHEP 05 (2017) 027 [arXiv:1611.08407] [INSPIRE].

[5] N. Arkani-Hamed and J. Maldacena, Cosmological Collider Physics, arXiv:1503.08043 [INSPIRE].

[6] Bateman Manuscript Project, H. Bateman and A. Erdélyi, Spherical and hyperspherical harmonic polynomials, in Higher Transcendental Functions Volume II, McGraw-Hill Book Company, Inc., chapter XI (1953).

[7] N.J. Vilenkin, Special Functions and the Theory of Group Representations, American Mathematical Society (1968).

[8] K. Atkinson and W. Han, Spherical Harmonics and Approximations on the Unit Sphere: An Introduction, Springer (2010).

[9] S. Ferrara, A.F. Grillo, R. Gatto and G. Parisi, Analyticity properties and asymptotic expansions of conformal covariant green's functions, Nuovo Cim. A 19 (1974) 667 [INSPIRE].

[10] G.M. Sotkov and R.P. Zaikov, Conformal Invariant Two Point and Three Point Functions for Fields with Arbitrary Spin, Rept. Math. Phys. 12 (1977) 375 [INSPIRE].

\footnotetext{
${ }^{12}$ Here we used the fact that any spherical harmonic on the unit sphere $S_{D-1}$ is the restriction of a harmonic polynomial in $\mathbb{R}^{D}$ (i.e. a homogeneous polynomial annihilated by the Laplacian in $\mathbb{R}^{D}$ ) to $S_{D-1}$. The spin of the spherical harmonic is the degree of homogeneity of the corresponding harmonic polynomial.
} 
[11] G.M. Sotkov and R.P. Zaikov, On the Structure of the Conformal Covariant N Point Functions, Rept. Math. Phys. 19 (1984) 335 [inSPIRE].

[12] I. Antoniadis, P.O. Mazur and E. Mottola, Conformal Invariance, Dark Energy and CMB Non-Gaussianity, JCAP 09 (2012) 024 [arXiv:1103.4164] [INSPIRE].

[13] J.M. Maldacena and G.L. Pimentel, On graviton non-Gaussianities during inflation, JHEP 09 (2011) 045 [arXiv: 1104.2846] [INSPIRE].

[14] C. Corianò, L. Delle Rose and M. Serino, Three and Four Point Functions of Stress Energy Tensors in D = 3 for the Analysis of Cosmological Non-Gaussianities, JHEP 12 (2012) 090 [arXiv: 1210.0136] [INSPIRE].

[15] D. Chowdhury, S. Raju, S. Sachdev, A. Singh and P. Strack, Multipoint correlators of conformal field theories: implications for quantum critical transport, Phys. Rev. B 87 (2013) 085138 [arXiv: 1210 . 5247] [INSPIRE].

[16] A. Bzowski, P. McFadden and K. Skenderis, Holography for inflation using conformal perturbation theory, JHEP 04 (2013) 047 [arXiv: 1211.4550] [INSPIRE].

[17] I. Mata, S. Raju and S. Trivedi, CMB from CFT, JHEP 07 (2013) 015 [arXiv:1211.5482] [INSPIRE].

[18] C. Corianò, L. Delle Rose, E. Mottola and M. Serino, Solving the Conformal Constraints for Scalar Operators in Momentum Space and the Evaluation of Feynman's Master Integrals, JHEP 07 (2013) 011 [arXiv: 1304.6944] [INSPIRE].

[19] A. Bzowski, P. McFadden and K. Skenderis, Implications of conformal invariance in momentum space, JHEP 03 (2014) 111 [arXiv:1304.7760] [INSPIRE].

[20] Y. Huh, P. Strack and S. Sachdev, Conserved current correlators of conformal field theories in 2+1 dimensions, Phys. Rev. B 88 (2013) 155109 [Erratum ibid. B 90 (2014) 199902] [arXiv: 1307.6863] [INSPIRE].

[21] A. Ghosh, N. Kundu, S. Raju and S.P. Trivedi, Conformal Invariance and the Four Point Scalar Correlator in Slow-Roll Inflation, JHEP 07 (2014) 011 [arXiv:1401.1426] [INSPIRE].

[22] N. Kundu, A. Shukla and S.P. Trivedi, Constraints from Conformal Symmetry on the Three Point Scalar Correlator in Inflation, JHEP 04 (2015) 061 [arXiv:1410.2606] [INSPIRE].

[23] N. Kundu, A. Shukla and S.P. Trivedi, Ward Identities for Scale and Special Conformal Transformations in Inflation, JHEP 01 (2016) 046 [arXiv:1507.06017] [INSPIRE].

[24] K. Sen and A. Sinha, On critical exponents without Feynman diagrams, J. Phys. A 49 (2016) 445401 [arXiv:1510.07770] [INSPIRE].

[25] A. Bzowski, P. McFadden and K. Skenderis, Scalar 3-point functions in CFT: renormalisation, $\beta$-functions and anomalies, JHEP 03 (2016) 066 [arXiv:1510.08442] [INSPIRE].

[26] A. Bzowski, P. McFadden and K. Skenderis, Evaluation of conformal integrals, JHEP 02 (2016) 068 [arXiv: 1511.02357] [INSPIRE].

[27] V.P.J. Jacobs, P. Betzios, U. Gürsoy and H.T.C. Stoof, Electromagnetic response of interacting Weyl semimetals, Phys. Rev. B 93 (2016) 195104 [arXiv:1512.04883] [InSPIRE].

[28] R.C. Myers, T. Sierens and W. Witczak-Krempa, A Holographic Model for Quantum Critical Responses, JHEP 05 (2016) 073 [arXiv: 1602.05599] [INSPIRE]. 
[29] A. Lucas, S. Gazit, D. Podolsky and W. Witczak-Krempa, Dynamical response near quantum critical points, Phys. Rev. Lett. 118 (2017) 056601 [arXiv: 1608.02586] [INSPIRE].

[30] A. Lucas, T. Sierens and W. Witczak-Krempa, Quantum critical response: from conformal perturbation theory to holography, JHEP 07 (2017) 149 [arXiv: 1704.05461] [INSPIRE].

[31] A. Bzowski, P. McFadden and K. Skenderis, Renormalised 3-point functions of stress tensors and conserved currents in CFT, JHEP 11 (2018) 153 [arXiv:1711.09105] [INSPIRE].

[32] C. Corianò and M.M. Maglio, Exact Correlators from Conformal Ward Identities in Momentum Space and the Perturbative TJJ Vertex, Nucl. Phys. B 938 (2019) 440 [arXiv: 1802.07675] [INSPIRE].

[33] A. Bzowski, P. McFadden and K. Skenderis, Renormalised CFT 3-point functions of scalars, currents and stress tensors, JHEP 11 (2018) 159 [arXiv: 1805.12100] [INSPIRE].

[34] M. Gillioz, Momentum-space conformal blocks on the light cone, JHEP 10 (2018) 125 [arXiv: 1807.07003] [INSPIRE].

[35] C. Corianò and M.M. Maglio, The general 3-graviton vertex (TTT) of conformal field theories in momentum space in $d=4$, Nucl. Phys. B 937 (2018) 56 [arXiv:1808.10221] [InSPIRE].

[36] S. Albayrak and S. Kharel, Towards the higher point holographic momentum space amplitudes, JHEP 02 (2019) 040 [arXiv:1810.12459] [INSPIRE].

[37] N. Arkani-Hamed, D. Baumann, H. Lee and G.L. Pimentel, The Cosmological Bootstrap: Inflationary Correlators from Symmetries and Singularities, arXiv:1811.00024 [INSPIRE].

[38] E. Skvortsov, Light-Front Bootstrap for Chern-Simons Matter Theories, JHEP 06 (2019) 058 [arXiv: 1811.12333] [INSPIRE].

[39] J.A. Farrow, A.E. Lipstein and P. McFadden, Double copy structure of CFT correlators, JHEP 02 (2019) 130 [arXiv: 1812.11129] [INSPIRE].

[40] H. Isono, T. Noumi and T. Takeuchi, Momentum space conformal three-point functions of conserved currents and a general spinning operator, JHEP 05 (2019) 057 [arXiv: 1903.01110] [INSPIRE].

[41] C. Corianò and M.M. Maglio, On Some Hypergeometric Solutions of the Conformal Ward Identities of Scalar 4-point Functions in Momentum Space, JHEP 09 (2019) 107 [arXiv: 1903.05047] [INSPIRE].

[42] C. Sleight, A Mellin Space Approach to Cosmological Correlators, arXiv:1906.12302 [INSPIRE].

[43] C. Sleight and M. Taronna, Bootstrapping Inflationary Correlators in Mellin Space, arXiv: 1907.01143 [INSPIRE].

[44] S. Albayrak and S. Kharel, Towards the higher point holographic momentum space amplitudes II: Gravitons, arXiv:1908.01835 [INSPIRE].

[45] V. Bargmann and I.T. Todorov, Spaces of Analytic Functions on a Complex Cone as Carries for the Symmetric Tensor Representations of SO(N), J. Math. Phys. 18 (1977) 1141 [INSPIRE].

[46] M.S. Costa, J. Penedones, D. Poland and S. Rychkov, Spinning Conformal Correlators, JHEP 11 (2011) 071 [arXiv:1107.3554] [INSPIRE].

[47] I.S. Gradshteyn and I.M. Ryzhik, Table of Integrals, Series and Products, Seventh Edition, Elsevier, Academic Press (2007). 